\begin{tabular}{|c|l|}
\hline Title & Ligand-dependent transcription of estrogen receptor a is mediated by the ubiquitin ligase EFP \\
\hline Author(s) & $\begin{array}{l}\text { Nakajima, A yako; Maruy ama, Satoru; Bohgaki, Miyuki; Miyajima, Naoto; Tsukiyama, Tadasuke; Sakuragi, Noriaki; } \\
\text { Hatakeyama, Shigetsugu }\end{array}$ \\
\hline Citation & $\begin{array}{l}\text { Biochemical and Biophysical Research Communications, 357(1), 245-251 } \\
\text { https://doi.org/10.1016/.bbrc.2007.03.134 }\end{array}$ \\
\hline Issue Date & $2007-05-25$ \\
\hline Doc URL & http://hdl.handle.net/2115/24261 \\
\hline Type & article (author version) \\
\hline File Information & BBRC357-1.pdf \\
\hline
\end{tabular}

Instructions for use 


\title{
Ligand-dependent transcription of estrogen receptor $\alpha$ is mediated by the ubiquitin ligase EFP
}

\author{
Ayako Nakajima a,b ${ }^{\text {a, }}$ Satoru Maruyama ${ }^{a}$, Miyuki Bohgaki ${ }^{a}$, Naoto Miyajima ${ }^{a}$, \\ Tadasuke Tsukiyama ${ }^{a}$, Noriaki Sakuragi ${ }^{\mathrm{b}}$ and Shigetsugu Hatakeyama ${ }^{\mathrm{a}, *}$ \\ ${ }^{a}$ Department of Molecular Biochemistry and ${ }^{b}$ Department of Gynecology, Hokkaido \\ University Graduate School of Medicine, N15, W7, Kita-ku, Sapporo, Hokkaido \\ 060-8638, Japan. \\ * Corresponding authors. Shigetsugu Hatakeyama, Department of Molecular \\ Biochemistry, Hokkaido University Graduate School of Medicine, N15, W7, Kita-ku, \\ Sapporo, Hokkaido 060-8638, Japan. Phone: +81 11706 5899; Fax: +81 11706 5169; \\ E-mail address: hatas@med.hokudai.ac.jp.
}

Key words: estrogen receptor; estrogen-responsive finger protein; transcription; ubiquitylation 


\section{Abstract}

Estrogen-mediated ubiquitylation and subsequent degradation of the estrogen receptor $\alpha(E R \alpha)$ appears to be involved in the transcriptional activity of ER $\alpha$. We show that the estrogen-responsive finger protein (EFP) interacts with and ubiquitylates $\mathrm{ER} \alpha$. EFP promoted the ubiquitylation of $\mathrm{ER} \alpha$ in vitro and in vivo and consequently promoted the degradation of ER $\alpha$. The interaction between EFP and $\mathrm{ER} \alpha$ was greatly enhanced in the presence of estrogen. The action of EFP on ER $\alpha$ in the presence of estrogen resulted in a robust interaction between $\mathrm{ER} \alpha$ and Tip60, one of the transcriptional coactivators, leading to activation of $\mathrm{ER} \alpha$ transcriptional activity. However, a dominant negative mutant of EFP lacking the RING domain prolonged the half-life of ER $\alpha$ and inhibited the transcription by ER $\alpha$. Our results indicate that EFP functions as a cofactor for ER $\alpha$-mediated transcription, thus suggesting that ER $\alpha$-mediated transcription is closely linked to the ubiquitylation of ER $\alpha$. 


\section{Introduction}

Estrogen is a hormone that plays crucial roles in cell growth and differentiation and in regulation of the female reproductive system. The multifunctional effects of estrogen are elicited through interaction with the estrogen receptor (ER). ER $\alpha$ and ER $\beta$ belong to the steroid hormone superfamily of nuclear receptors, which act as ligand-dependent transcriptional factors [1]. Native unliganded ER $\alpha$ occurs in complex with the molecular chaperone Hsp90 and cochaperones, contributing to maintenance of the receptor stability. When bound to estrogen, ER $\alpha$ undergoes conformational changes, dissociations from heat shock proteins, a receptor dimerization, DNA bindings at the estrogen responsive element (ERE) of the target, interactions with coactivators, and the subsequent recruitment of transcription factors to be formed by either up-regulation or down-regulation of target gene transcription [2]. A number of coactivators of nuclear receptors have been identified, including members of the p160 family (SRC-1, TIF-2 and SRC-3), p300/CBP and Tip60, which exhibit histone acetyltransferase activities and recruit components of the general transcription machinery $[3,4]$.

Furthermore, the involvement of ubiquitin-proteasome components in ER $\alpha$-mediated transcription has been suggested by the results of studies utilizing proteasome inhibitors [5]. Upon treatment of cells with $17 \beta$-estradiol, ubiquitylation of $\mathrm{ER} \alpha$ is enhanced and the degradation of $\mathrm{ER} \alpha$ by a proteasome-dependent proteolytic pathway is required for efficient ER $\alpha$ transactivation [6]. Ubiquitin conjugation is

catalyzed by enzymes designated as ubiquitin-activating enzyme (E1), 
ubiquitin-conjugating enzyme (E2), and ubiquitin ligase (E3). The resulting covalent ubiquitin ligations form polyubiquitylated conjugates that are rapidly detected and degraded by $26 \mathrm{~S}$ proteasome. E3 has so far been shown to include members of the

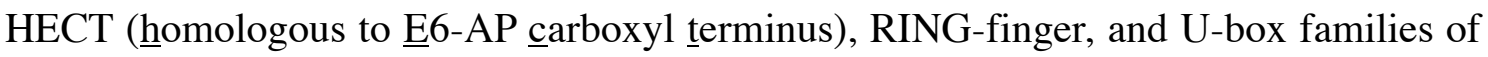
proteins [7-9].

Here we provide evidence that the estrogen-responsive finger protein (EFP) promotes the ubiquitylation and proteasome-mediated degradation of liganded ER $\alpha$. We show that EFP binds to and ubiquitylates ER $\alpha$ to activate transcription, suggesting that EFP may function as a cofactor for ER $\alpha$-induced transcription. 


\section{Materials and methods}

Cell culture. HEK293T and HeLa cells (ATCC) were cultured under an atmosphere of $5 \% \mathrm{CO}_{2}$ at $37^{\circ} \mathrm{C}$ in Dulbecco's modified Eagle's medium (Sigma) supplemented with $10 \%$ fetal bovine serum (Invitrogen). MCF-7 cells (ATCC) were cultured under the same conditions with $0.01 \mathrm{mg} / \mathrm{ml}$ insulin (Sigma).

Cloning of cDNAs and plasmid construction. Human EFP and ER $\alpha$ cDNAs were amplified from MCF-7 cDNA by the polymerase chain reaction (PCR) with KOD plus (TOYOBO) using primers 5'-GCCATGGCAGAGCTGTGCCCCCTG-3' (EFP-sense),

5'-TGCCTACTTGGGGGAGCAGATGGA-3' (EFP-antisense), 5'-ATGACCATGACCCTCCACACCAAA-3' (ER $\alpha$-sense), and 5'-TCAGACTGTGGCAGGGAAACCCTC-3' (ER $\alpha$-antisense). The amplified fragments were subcloned into pBluescript II SK ${ }^{+}$(Stratagene). The FLAG-tagged EFP cDNA and FLAG- or HA-tagged ER $\alpha$ cDNA were then subcloned into pCGN, pcDNA3 (Invitrogen), pFastBacHT (Invitrogen), pBTM116 and pACT2 (Clontech). Site-specific mutagenesis was performed with a Quick Change kit (Stratagene). Human Tip60 cDNA was obtained from Dr. Ikura (Tohoku University). The deleted mutants of ER $\alpha$ cDNA were generated by PCR.

Baculovirus expression system. The plasmids pFastBacHT containing the relevant cDNAs were subjected to recombination with the baculoviral genome in DH10BAC, and the resulting recombinant viral genome was introduced into Sf9 cells by transfection in order to generate recombinant baculoviruses. 
Transfection, immunoprecipitation, and immunoblot analysis. HEK293T cells were transfected by the calcium phosphate method [9]. Immunoblot analysis was performed with the following primary antibodies: anti-c-Myc (1 $\mu \mathrm{g} / \mathrm{ml}$; 9E10, Covance), anti-FLAG (1 $\mu \mathrm{g} / \mathrm{ml}$; M2 or M5, Sigma), anti-HA (1 $\mu \mathrm{g} / \mathrm{ml}$; HA.11/16B12, Babco), anti-hexa-histidine (1 $\mu \mathrm{g} / \mathrm{ml} ; \mathrm{H}-15$, Santa Cruz), anti-Hsp90 (1 $\mu \mathrm{g} / \mathrm{ml} ; 68$, TDL), anti-EFP (1 $\mu \mathrm{g} / \mathrm{ml} ; 2$, TDL), anti-ubiquitin (1 $\mu \mathrm{g} / \mathrm{ml}$; P4D1, Santa Cruz) and anti-ER $\alpha$ (1 $\mu \mathrm{g} / \mathrm{ml} ; \mathrm{H}-184$, Santa Cruz).

In vitro pull-down assay. Recombinant $\mathrm{His}_{6}$-EFP $(0.5 \mu \mathrm{g})$ and recombinant $\mathrm{His}_{6}$-ER $\alpha(0.5 \mu \mathrm{g})$ were mixed in $50 \mathrm{mM}$ Tris- $\mathrm{HCl}(\mathrm{pH} 7.4), 150 \mathrm{mM} \mathrm{NaCl}$, and $1 \%$ Nonidet P-40 for $1 \mathrm{~h}$ at $4^{\circ} \mathrm{C}$. The binding mixtures were incubated with anti-EFP antibody for $1 \mathrm{~h}$ at $4^{\circ} \mathrm{C}$. Protein G-Sepharose, which was equilibrated with the same solution, was added to the mixture and then rotated for $1 \mathrm{~h}$ at $4{ }^{\circ} \mathrm{C}$. The resin was separated by centrifugation, washed five times with lysis buffer, and then boiled in SDS sample buffer. Immunoblot analysis was performed with anti-ER $\alpha$ antibody.

$\beta$-galactosidase assay using yeast two-hybrid system. $\beta$-galactosidase assays were performed as previously described [10]. Incubations were allowed to proceed for $5 \mathrm{~h}$ at $30^{\circ} \mathrm{C}$ and then the $\mathrm{OD}_{420}$ was taken to calculate the $\beta$-galactosidase activity using the following equation: $1000 \times \mathrm{OD}_{420} /\left(\mathrm{t} \times \mathrm{V} \times \mathrm{OD}_{600}\right)$, where $\mathrm{t}$ is the elapsed time (min) and $\mathrm{V}$ is $0.1 \mathrm{ml} \mathrm{x}$ concentration factor (concentration factor $=5$ in this experiment).

In vitro ubiquitylation assay. An in vitro ubiquitylation assay was performed as previously described. Reaction mixtures were incubated for $2 \mathrm{~h}$ at $30^{\circ} \mathrm{C}$ and then subjected to immunoblot analysis with a mouse monoclonal antibody to ubiquitin or 
$\mathrm{ER} \alpha[11]$.

Pulse-chase experiments. Transfected HEK293T cells were metabolically labeled with Trans ${ }^{35} \mathrm{~S}$ (ICN, PerkinElmer) at a concentration of $100 \mathrm{mCi} / \mathrm{ml}$ for $1 \mathrm{~h}$ and then $17 \beta$-estradiol $\left(10^{-8} \mathrm{M}\right.$, SIGMA) was added, and this was then chased. Cell lysates were immunoprecipitated with anti-HA antibody followed by purification with protein G-Sepharose, separated by SDS-PAGE, and exposed and quantified by BAS-2000 (Fuji Film, Kanagawa, Japan).

Luciferase assay. MCF-7 cells were cultured for $48 \mathrm{~h}$ in phenol red-free MEM containing dextran-coated charcoal-treated 10\% FCS (Hyclone) before transfection. $17 \beta$-estradiol $\left(10^{-8} \mathrm{M}\right)$ was added $48 \mathrm{~h}$ after transfection, and then after $12 \mathrm{~h}$ cells were assayed for luciferase activity with a Dual-Luciferase Reporter Assay System (Promega). 


\section{Results}

Interaction of EFP with ER $\alpha$

The Efp gene has been reported to have an ERE, and its mRNA is actually induced by estrogen treatment [12]. We investigated whether EFP physically interacts with ER $\alpha$. We expressed FLAG-tagged EFP, together with/without hemagglutinin epitope (HA)-tagged ER $\alpha$, in HEK293T cells. Cell lysates were subjected to immunoprecipitation with an antibody to FLAG, and the resulting precipitates were subjected to immunoblot analysis with an antibody to HA or FLAG. HA-ER $\alpha$ was co-precipitated by the antibody to FLAG in the presence of FLAG-EFP (Fig. 1A). To examine the interaction by an in vitro pull down assay, we generated and purified recombinant hexahistidine epitope $\left(\mathrm{His}_{6}\right)$-tagged EFP and ER. The recombinant $\mathrm{His}_{6}$-EFP was mixed with $\mathrm{His}_{6}$-ER $\alpha$ and then immunoprecipitated with an antibody to EFP, and the resulting precipitates were subjected to immunoblot analysis with an antibody to $\mathrm{ER} \alpha$ or EFP. $\mathrm{His}_{6}$-ER $\alpha$ was selectively co-precipitated by the antibody to EFP in the presence of $\mathrm{His}_{6}$-EFP (Fig. 1B). These results suggest that EFP specifically interacts with ER $\alpha$. To quantify the affinity between EFP and ER $\alpha$ in the presence or absence of estrogen (17 $\beta$-estradiol), we used a yeast two-hybrid system. EFP interacted weakly with ER $\alpha$ in a yeast two-hybrid assay in the absence of estrogen but interacted strongly with ER $\alpha$ in the presence of estrogen (Fig. 1C). Next we examined the ligand dependency of this interaction by using the mammalian cell line MCF-7 that 
endogenously expressed ER $\alpha$. It has been reported that $17 \beta$-estradiol causes proteasome-dependent degradation of ER $\alpha$ [13]. Thus, we investigated whether endogenous EFP interacts with endogenous ER $\alpha$ in MCF-7 cells in the presence of the proteasome inhibitor MG132. A small amount of ER $\alpha$ was detected in the immunoprecipitation performed with an anti-EFP antibody in the presence of $17 \beta$-estradiol but not in the immunoprecipitation with an anti-HA antibody as a negative control (Fig. 1D). The combination of $17 \beta$-estradiol and MG132 more greatly enhanced the endogenous interactions in vivo (Fig. 1D). These results suggest that estrogen contributes to the interaction between EFP and ER $\alpha$ in MCF-7 cells.

To identify the region of ER $\alpha$ responsible for the interaction with EFP, a series of ER $\alpha$ deletion derivatives were generated (Fig. 1E). Deletion of the N-terminal 296 amino acids (ER $\alpha(297-595))$ did not affect the binding to EFP, whereas the deletion of C-terminal 255 amino acids $(\mathrm{ER} \alpha(1-340))$ abolished the binding to EFP. The region containing amino acids 297-534 of ER $\alpha$, not comprising the coactivator binding region in AF-2, is required for the interaction with EFP (Fig. 1F).

Ubiquitin ligase activity of EFP

To determine whether EFP has an E3 ligase activity, we performed in vitro ubiquitylation assays. Immunoblot analysis of the reaction mixtures with antibodies to ubiquitin and EFP revealed the ubiquitylation of $\mathrm{His}_{6}$-EFP only in the presence of E1, E2 (UbcH5C), ubiquitin, ATP, and EFP itself (Fig. 2A). The lack of any of these 
components prevented self-ubiquitylation of EFP. EFP was thus shown to be a bona fide E3 ligase.

To further investigate the region of EFP responsible for ubiquitylation, a deletion mutant lacking the RING domain ( $\triangle$ RING) and a RING domain mutant in which the first cysteine was substituted by alanine (C8A) were generated (Fig. 2B). In vitro ubiquitylation assays indicated that an intact RING domain is indispensable for E3 ligase activity of EFP (Fig. 2C).

To analyze the class of polyubiquitin chains supported by E3 ligase activity of EFP, three mutant ubiquitins in which lysine was substituted by arginine at position 48 and/or 63, namely UbK48R, UbK63R and UbK48/63R, were purified. According to in vitro ubiquitylation assays, EFP underwent polyubiquitylation with UbK63R but not with UbK48R or UbK48/63R, implying that EFP predominantly uses the lysine at position 48 on ubiquitin for the formation of a polyubiquitylation chain (Fig. 2D and E). To further confirm polyubiquitylation of EFP through K48-based chains, recombinant proteins of mutants $\mathrm{UbK} 48$ and UbK63, each having one lysine residue at positions 48 and 63, respectively, and all other lysines substituted by arginine, were generated. EFP mediated the polyubiquitylation by UbK48 but not by UbK63, indicating the lysine at position 48 on ubiquitin is sufficient for the formation of a polyubiquitylation chain by EFP (Fig. 2E).

EFP ubiquitylates ER $\alpha$ in vitro and in vivo 
To determine whether EFP ubiquitylates ER $\alpha$, we assayed E3 ligase activity of His $_{6}$-EFP against $\mathrm{His}_{6}$-ER $\alpha$. Polyubiquitylation of $\mathrm{His}_{6}$-ER $\alpha$ was observed only in the additional presence of the $\mathrm{His}_{6}$-EFP (Fig. 3A). However, polyubiquitylation of ER $\alpha$ was not detected in the presence of $\operatorname{EFP}(\mathrm{C} 8 \mathrm{~A})$ (Fig. 3B). To further investigate the ubiquitylation of ER $\alpha$ in cells by in vivo ubiquitylation assay, FLAG-ER $\alpha$, EFP and HA-ubiquitin were expressed in HEK293T cells. FLAG-ER $\alpha$ was ubiquitylated extensively when cotransfected with EFP (Fig. 3C).

To examine the possible effect of EFP on the degradation of ER $\alpha$ in vivo, we transfected HEK293T cells with vectors for HA-ER $\alpha$ and for FLAG-EFP or FLAG-EFP $(\Delta \mathrm{RING})$ as a dominant negative form. Pulse-chase analysis in the presence of estrogen revealed that the expression of EFP exhibits the degradation of ER $\alpha$, whereas overexpression of $\operatorname{EFP}(\Delta \mathrm{RING})$ delays the degradation of ER $\alpha$ (Fig. 3D and E). These results thus suggest that EFP contributes to the turnover of ER $\alpha$ in cells.

EFP enhances activation of transcription by ER $\alpha$

To examine the effect of EFP on ER $\alpha$-induced transcription in MCF-7 cells, we measured the relative luciferase activity of MCF-7 cells transfected with an ERE reporter construct with basal HSV-thymidine kinase gene promoter (pTK-ERE-Luc). The relative luciferase activity of cells with the addition of $17 \beta$-estradiol or with the overexpression of EFP was increased compared with that of mock-transfected cells. The combination of the addition of $17 \beta$-estradiol with the overexpression of EFP resulted in 
a further increase of luciferase activity, whereas $\operatorname{EFP}(\Delta \mathrm{RING})$ inhibited the activity even in the presence of $17 \beta$-estradiol, suggesting that EFP acts as a positive regulator for transcriptional activation by ER $\alpha$ (Fig. 4A).

Transcriptional regulation by ER $\alpha$ involves protein-protein interactions among the receptor and its associated coactivators, including Tip60. It is known that interaction between ER $\alpha$ and coactivators enhances ER $\alpha$-mediated transcriptional initiation. To explore the interdependence between EFP and coactivators in activation of the ERE promoter, we examined the effect of EFP on Tip60-induced transcription in MCF-7 cells. Overexpression of Tip60 activated the ERE promoter in the presence of $17 \beta$-estradiol, and the combination of EFP and Tip60 resulted in greater activation of the ERE promoter (Fig. 4B). Similar results were obtained for another coactivator, p300 (data not shown). These findings suggest that EFP assists the ER $\alpha$-mediated transcription together with coactivators.

To clarify the relationship between the transcriptional activity and the ubiquitylation, we examined the level of physical interaction between $\mathrm{ER} \alpha$ and coactivators, especially Tip60. Physical interaction between ER $\alpha$ and Tip60 was detectable by coimmunoprecipitation in the presence of $17 \beta$-estradiol (Fig. 4C). We thus expressed Myc-EFP(WT/C8A), HA-ER $\alpha$ and FLAG-Tip60 in HEK293T cells, followed by immunoprecipitation with an antibody to FLAG and detection with an anti-HA antibody. The interaction of ER $\alpha$ with Tip60 was more greatly enhanced in cells expressing EFP(WT), whereas the interaction was reduced in cells expressing the dominant negative $\mathrm{EFP}(\mathrm{C} 8 \mathrm{~A})$, indicating that EFP is likely to promote the interaction between 
ER $\alpha$ and Tip60 (Fig. 4D). 


\section{Discussion}

Here we have demonstrated that EFP directly interacts with ER $\alpha$ in an estrogen-dependent manner and ubiquitylates ER $\alpha$ and that the ubiquitylation by EFP regulates the transcription of ER $\alpha$ including the interaction with coactivators, followed by the degradation of ER $\alpha$. Previous studies indicated that the inhibition of proteasomal degradation markedly increased estrogen-dependent transcription on an $E R E$-responsive reporter in HeLa cells transfected with ER $\alpha$. Blocking ligand-induced degradation of $\mathrm{ER} \alpha$ by proteasome inhibitors resulted in prolonged simulation of ER-responsive gene transcription [14]. These observations suggest that proteasomal degradation of ER $\alpha$ leads to attenuation of transactivation but do not rule out the possibility of ubiquitylation to initiate the transcription of $\mathrm{ER} \alpha$. We have shown that EFP functions as a cofactor by recruiting coactivators that have histone acetyltransferase activities, such as Tip60. Previous studies have shown that transcriptional regulation by ER $\alpha$ involves interactions among the receptor, coactivators and RNA polymerase II. Tip60 is one of the nuclear receptor coactivators and enhances transactivation though liganded ER $\alpha$ in collaboration with other coactivators, p300/CBP [15]. It has been reported that Skp2 binds to c-Myc and thereby mediates its ubiquitylation. Skp2 also increases the transcriptional activity of c-Myc, suggesting that Skp2 is a transcriptional cofactor [16]. Moreover, it has been shown that HectH9-mediated ubiquitylation of Myc is required for transactivation of multiple target genes by recruitment of the coactivator p300 [17]. EFP-mediated ER $\alpha$ ubiquitylation may be crucial for recruitment of the transcriptional 
cofactors as well.

It has been shown that Efp-/- mice of both sexes were viable and fertile and that the uterus, which expressed a high level of ER $\alpha$ in wild-type mice, exhibited significant underdevelopment [18]. We have demonstrated that the transcriptional activation of ER $\alpha$ is enhanced by EFP. Therefore, these phenotypes of Efp-/- mice can be explained by the balance of interaction between EFP and ER $\alpha$.

Recently, another E3 ligase, CHIP, has been reported to induce ubiquitylation of unliganded $\mathrm{ER} \alpha$, and CHIP probably degrades unfolded and misfolded $\mathrm{ER} \alpha$ to maintain quality control of ER $\alpha$ in the cytoplasm [19]. The ubiquitylation of ER $\alpha$ by CHIP is independent of estrogen and serves for the degradation of misfolded ER $\alpha$. EFP ubiquitylates $\mathrm{ER} \alpha$ dependent on estrogen to up-regulate its transcriptional activity. These findings demonstrate that the role of EFP is different from that of CHIP with regards to the ubiquitylation of ER $\alpha$.

In breast and endometrial carcinomas which are classified as estrogen-responsive malignant tumors, estrogen is one of the significant factors with regard to anti-tumor therapies and their prognoses. Our findings suggest that the expression levels of EFP are correlated with the histological grade of an estrogen-responsive tumor, and results of large-scale clinicopathological analyses of EFP should contribute to the clinical knowledge of breast, ovarian or endometrial carcinomas. 


\section{Acknowledgements}

We thank T. Ikura, E. Ogawa and K. Morohashi for the plasmids. This work was supported in part by a research grant from Grant-in-Aid for Scientific Research on Priority Areas from the Ministry of Education, Culture, Sports, Science and Technology, the Osaka Cancer Research Foundation and The Akiyama Foundation. 


\section{References}

[1] D.J. Mangelsdorf, C. Thummel, M. Beato, P. Herrlich, G. Schutz, K. Umesono, B. Blumberg, P. Kastner, M. Mark, P. Chambon, R.M. Evans, The nuclear receptor superfamily: the second decade, Cell 83 (1995) 835-839.

[2] M.Y. Kim, S.J. Hsiao, W.L. Kraus, A role for coactivators and histone acetylation in estrogen receptor $\alpha$-mediated transcription initiation, EMBO J. 20 (2001) 6084-6094.

[3] M.E. Brady, D.M. Ozanne, L. Gaughan, I. Waite, S. Cook, D.E. Neal, C.N. Robson, Tip60 is a nuclear hormone receptor coactivator, J. Biol. Chem. 274 (1999) 17599-17604.

[4] C.K. Glass, M.G. Rosenfeld, The coregulator exchange in transcriptional functions of nuclear receptors, Gene. Dev, 14 (2000) 121-141.

[5] D.M. Lonard, Z. Nawaz, C.L. Smith, B.W. O'Malley, The 26S proteasome is required for estrogen receptor- $\alpha$ and coactivator turnover and for efficient estrogen receptor-alpha transactivation, Mol. Cell 5 (2000) 939-948.

[6] I. Laios, F. Journe, D. Nonclercq, D.S. Vidal, R.A. Toillon, G. Laurent, G. Leclercq, Role of the proteasome in the regulation of estrogen receptor $\alpha$ turnover and function in MCF-7 breast carcinoma cells, J. Steroid Biochem. Mol. Biol. 94 (2005) 347-359.

[7] J.M. Huibregtse, M. Scheffner, S. Beaudenon, P.M. Howley, A family of proteins structurally and functionally related to the E6-AP ubiquitin-protein ligase, Proc. Natl. Acad. Sci. USA 92 (1995) 2563-2567. 
[8] K.L. Lorick, J.P. Jensen, S. Fang, A.M. Ong, S. Hatakeyama, A.M. Weissman, RING fingers mediate ubiquitin-conjugating enzyme (E2)-dependent ubiquitination, Proc. Natl. Acad. Sci. USA 96 (1999) 11364-11369.

[9] S. Hatakeyama, M. Yada, M. Matsumoto, N. Ishida, K.I. Nakayama, U box proteins as a new family of ubiquitin-protein ligases, J. Biol. Chem. 276 (2001) 33111-33120.

[10] S. Hatakeyama, M. Matsumoto, M. Yada, K.I. Nakayama, Interaction of U-box-type ubiquitin-protein ligases (E3s) with molecular chaperones, Genes Cells 9 (2004) 533-548.

[11] S. Hatakeyama, M. Watanabe, Y. Fujii, K.I. Nakayama, Targeted destruction of c-Myc by an engineered ubiquitin ligase suppresses cell transformation and tumor formation, Cancer Res. 65 (2005) 7874-7879.

[12] S. Inoue, A. Orimo, T. Hosoi, S. Kondo, H. Toyoshima, T. Kondo, A. Ikegami, Y. Ouchi, H. Orimo, M. Muramatsu, Genomic binding-site cloning reveals an estrogen-responsive gene that encodes a RING finger protein, Proc. Natl. Acad. Sci. USA 90 (1993) 11117-11121.

[13] Z. Nawaz, D.M. Lonard, A.P. Dennis, C.L. Smith, B.W. O'Malley, Proteasome-dependent degradation of the human estrogen receptor, Proc. Natl. Acad. Sci. USA 96 (1999) 1858-1862.

[14] M. Fan, H. Nakshatri, K.P. Nephew, Inhibiting proteasomal proteolysis sustains estrogen receptor-alpha activation, Mol. Endocrinol. 18 (2004) 2603-2615.

[15] T. Ikura, V.V. Ogryzko, M. Grigoriev, R. Groisman, J. Wang, M. Horikoshi, R. Scully, J. Qin, Y. Nakatani, Involvement of the TIP60 histone acetylase complex in 
DNA repair and apoptosis, Cell 102 (2000) 463-473.

[16] N. von der Lehr, S. Johansson, S. Wu, F. Bahram, A. Castell, C. Cetinkaya, P. Hydbring, I. Weidung, K. Nakayama, K.I. Nakayama, O. Soderberg, T.K. Kerppola, L.G. Larsson, The F-box protein Skp2 participates in c-Myc proteosomal degradation and acts as a cofactor for c-Myc-regulated transcription, Mol. Cell 11 (2003) 1189-1200.

[17] S. Adhikary, F. Marinoni, A. Hock, E. Hulleman, N. Popov, R. Beier, S. Bernard, M. Quarto, M. Capra, S. Goettig, U. Kogel, M. Scheffner, K. Helin, M. Eilers, The ubiquitin ligase HectH9 regulates transcriptional activation by Myc and is essential for tumor cell proliferation, Cell 123 (2005) 409-421.

[18] A. Orimo, S. Inoue, O. Minowa, N. Tominaga, Y. Tomioka, M. Sato, J. Kuno, H. Hiroi, Y. Shimizu, M. Suzuki, T. Noda, M. Muramatsu, Underdeveloped uterus and reduced estrogen responsiveness in mice with disruption of the estrogen-responsive finger protein gene, which is a direct target of estrogen receptor alpha, Proc. Natl. Acad. Sci. USA 96 (1999) 12027-12032.

[19] Y. Tateishi, Y. Kawabe, T. Chiba, S. Murata, K. Ichikawa, A. Murayama, K. Tanaka, T. Baba, S. Kato, J. Yanagisawa, Ligand-dependent switching of ubiquitin-proteasome pathways for estrogen receptor, EMBO J. 23 (2004) 4813-4823. 


\section{Figure Legends}

Fig. 1. EFP binds to ER $\alpha$ in vivo and in vitro. (A) In vivo binding assay between EFP and ER $\alpha$. FLAG-EFP and HA-ER $\alpha$ expression vectors were transfected into HEK293T cells. Cell lysates (WCL) were subjected to immunoprecipitation (IP) with antibodies to FLAG, immunoblotted (IB), and probed with antibodies to HA or FLAG. (B) In vitro pull-down assay between EFP and ER $\alpha$. Recombinant $\mathrm{His}_{6}$-EFP and $\mathrm{His}_{6}$-ER $\alpha$ were mixed in the combination as indicated. The reaction mixture was immunoprecipitated with anti-EFP antibody and immunoblotted with anti-ER $\alpha$. (C) L40 yeast cells were cotransfected with pBTM116-EFP and pACT2-ER $\alpha$, and then three independent clones were selected, grown with $17 \beta$-estradiol $\left(10^{-7} \mathrm{M}\right)$ or with ethanol as a mock, and harvested. The $\beta$-galactosidase activities of cell lysates were calculated in ONPG assays. (D) Interaction between endogenous EFP and ER $\alpha$ in MCF-7 cells. Cells were incubated with $17 \beta$-estradiol $\left(10^{-8} \mathrm{M}\right)$ and/or MG132 $\left(10^{-5} \mathrm{M}\right)$ for $3 \mathrm{~h}$, and the cell lysates were immunoprecipitated with anti-EFP antibody or with anti-HA antibody as a control and then the resulting precipitates were subjected to immunoblot analysis with antibodies to EFP and ER $\alpha$. (E) Schematic representation of ER $\alpha$ deletion mutants. A/B, $\mathrm{N}$-terminal region including activation function 1; C, a conserved DNA binding domain, $\mathrm{D}$; a hinge region containing nuclear localization signal; $\mathrm{E} / \mathrm{F}$, C-terminal region including activation function 2. (F) The region of ER $\alpha$ which interacts with EFP. HEK293T cells were transfected with vectors for HA-EFP, and either full-length ER $\alpha$ or deleted mutants tagged with the FLAG epitope. Cell lysates were 
immunoprecipitated with anti-FLAG antibody, and then immunoblotted with anti-HA antibody.

Fig. 2. Ubiquitin ligase activity of EFP. (A) In vitro ubiquitylation assays were performed and the reaction mixtures were subjected to immunoblot analysis with antibodies to ubiquitin (upper panel) or to EFP (lower panel). (B) Schematic representation of the domain structures of $\mathrm{EFP}(\mathrm{WT}), \mathrm{EFP}(\triangle \mathrm{RING})$ and $\mathrm{EFP}(\mathrm{C} 8 \mathrm{~A})$. (C) Requirement of the RING domain in EFP for E3 ligase activity. Equimolar amounts of the indicated EFP derivative (WT, $\mathrm{C} 8 \mathrm{~A}$ or $\triangle \mathrm{RING}$ ) proteins were assayed for ubiquitylation activity in the presence of E1 and E2 (UbcH5C). (D) Specificity of lysine residue on ubiquitin for polyubiquitylation by EFP. Recombinant wild-type and mutant ubiquitins (UbK48R, UbK63R) were used for in vitro ubiquitylation assays. (E) Lysine 48 on ubiquitin is sufficient for polyubiquitylation by EFP. Recombinant wild-type and mutant ubiquitins (UbK48/63R, UbK48 and UbK63) were used for in vitro ubiquitylation assays.

Fig. 3. EFP mediates the ubiquitylation of $\mathrm{ER} \alpha$ in vitro and in vivo. (A) In vitro ubiquitylation assay for ER $\alpha$ by EFP. The reaction mixture was also subjected to immunoblot analysis with antibodies to ER $\alpha$ (upper panel) or to EFP (lower panel). (B) Requirement of the RING domain of EFP for ER $\alpha$. EFP derivatives (WT and C8A) were assayed for the polyubiquitylation of $\mathrm{ER} \alpha$ by in vitro ubiquitylation assays. (C) In vivo ubiquitylation assay for ER $\alpha$ with EFP. Expression vectors for EFP, FLAG-ER $\alpha$ 
and HA-Ub were transfected into HEK293T cells, and the cell lysates were immunoprecipitated with anti-FLAG antibody and then anti-HA immunoblot analysis was performed to detect the ubiquitylation of ER $\alpha$. (D) Pulse chase analysis of ER $\alpha$ with EFP. HEK293T cells that had been transfected with an expression plasmid encoding HA-ER $\alpha$ and either FLAG-EFP or FLAG-EFP( $\Delta$ RING) were radiolabeled with $\left[{ }^{35} \mathrm{~S}\right]$-methionine, immunoprecipitated with anti-HA antibody, and then subjected to SDS-PAGE and autoradiographed. (E) Quantification of the autoradiogram in Fig. 3D was performed by BAS-2000, and the amount of HA-ER $\alpha$ at $0 \mathrm{~h}$ was defined as $100 \%$.

Fig. 4. EFP enhances ER $\alpha$-mediated transcriptional activation. (A) MCF-7 cells were transfected with pTK-ERE-Luc, pRL-TK, plus FLAG-EFP(WT or $\Delta$ RING) with $10^{-8} \mathrm{M}$ $17 \beta$-estradiol or mock (ethanol). The luciferase activities represent the average of three independent transfections normalized with the internal control. (B) MCF-7 cells expressing FLAG-EFP or mock were transfected with pTK-ERE-Luc, pRL-TK, plus HA-Tip60 as indicated with $10^{-8} \mathrm{M} 17 \beta$-estradiol or mock (ethanol), and then the luciferase activities were measured. (C) FLAG-Tip60 and HA-ER $\alpha$ expression vectors were transfected into HEK293T cells. Cells incubated with or without $10^{-8} \mathrm{M}$ $17 \beta$-estradiol for $6 \mathrm{~h}$. Cell lysates were subjected to immunoprecipitation with antibodies to FLAG, immunoblotted, and probed with antibodies to HA or FLAG. (D) Expression vectors encoding FLAG-Tip60, HA-ER $\alpha$ and Myc-EFP(WT or C8A) were transfected into HEK293T cells, and then the cells were incubated with $10^{-8} \mathrm{M}$ 
$17 \beta$-estradiol for $6 \mathrm{~h}$. Cell lysates were subjected to immunoprecipitation with an antibody to FLAG, immunoblotted, and probed with antibodies to HA or FLAG. 
A

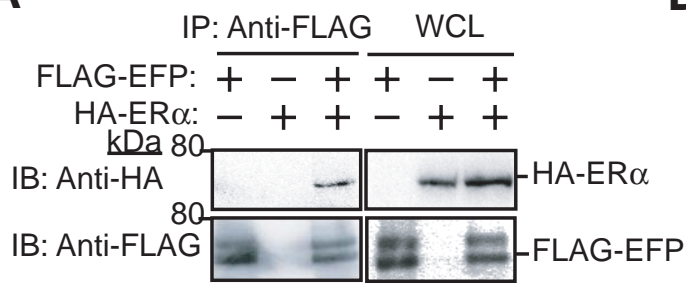

B

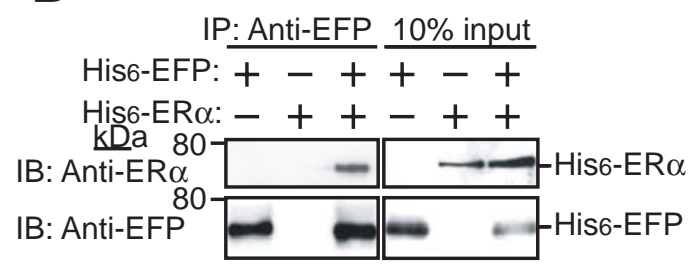

C

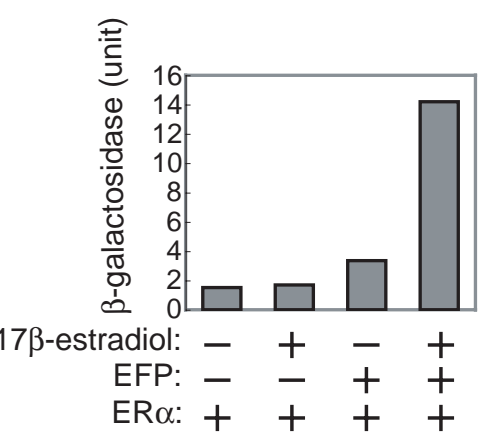

D

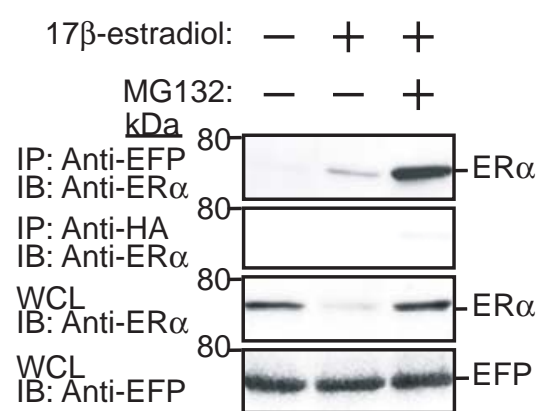

E
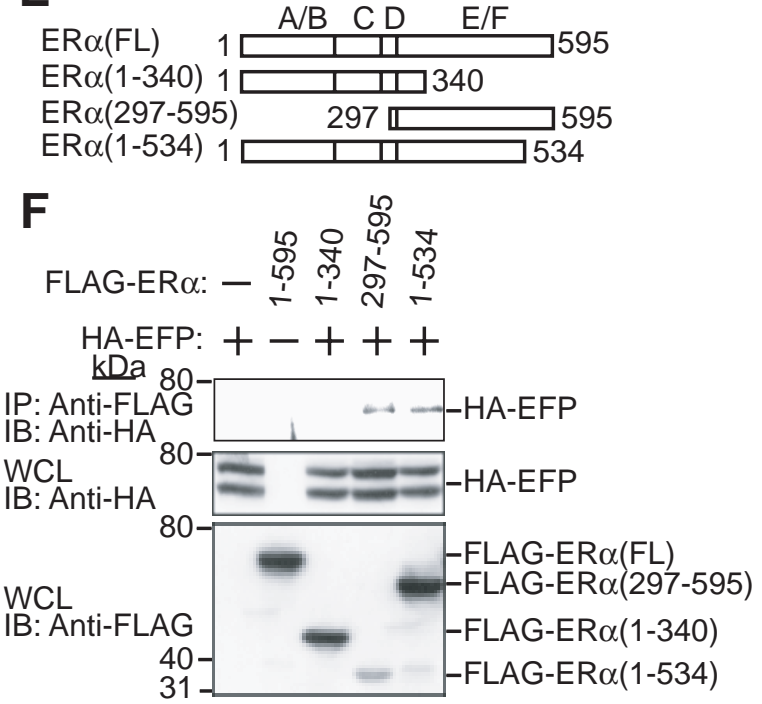


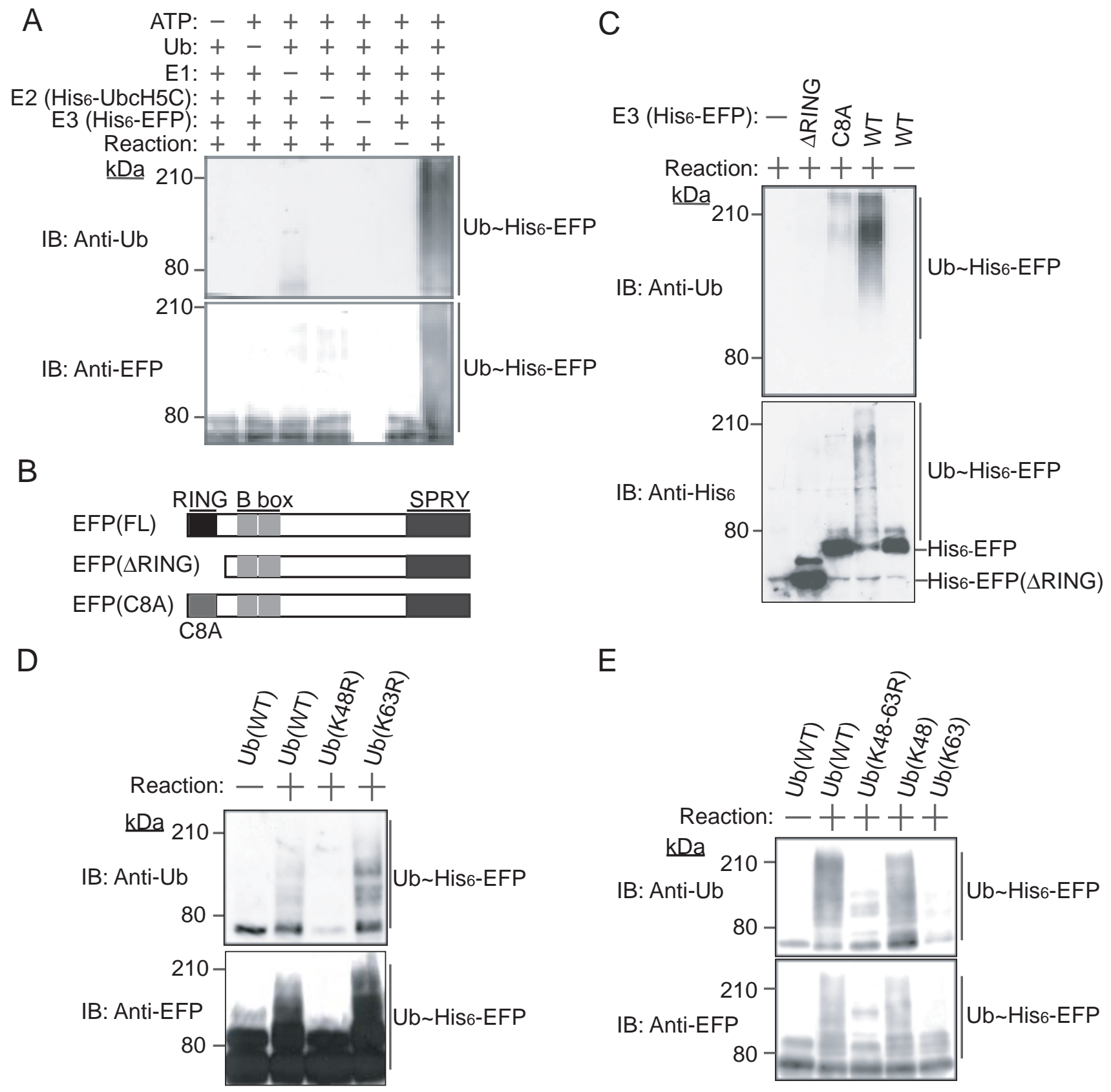


A

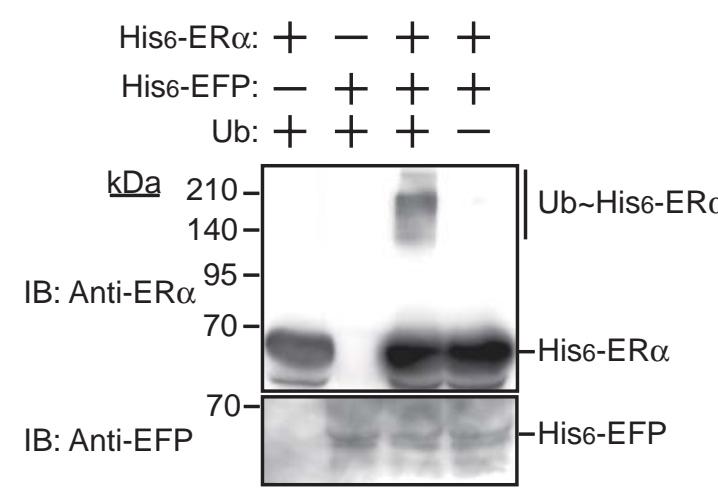

His6-ERo: +-++

Da

R $\alpha$

: Anti-EFP

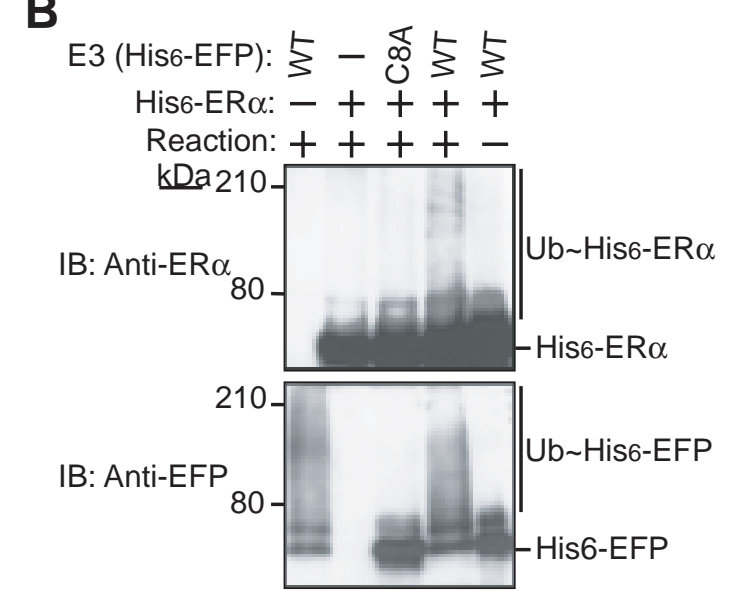

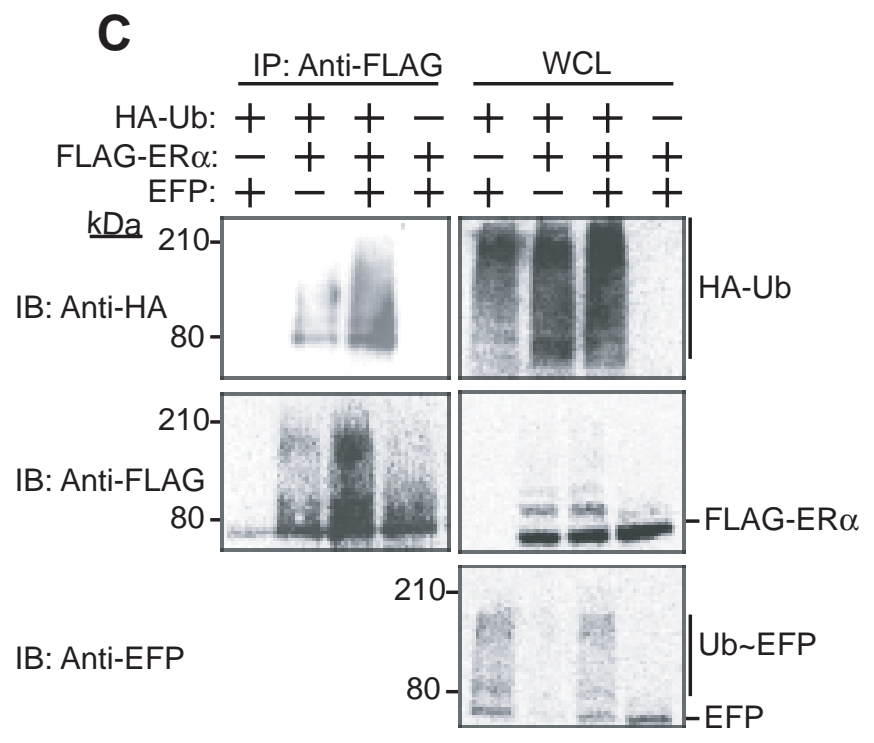

D

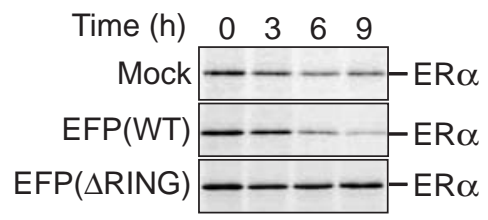

E

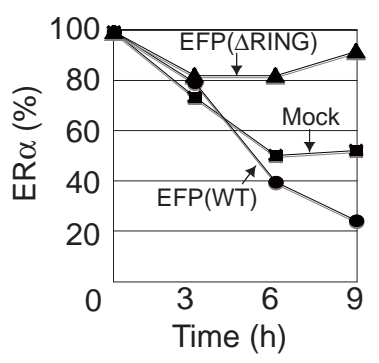


A

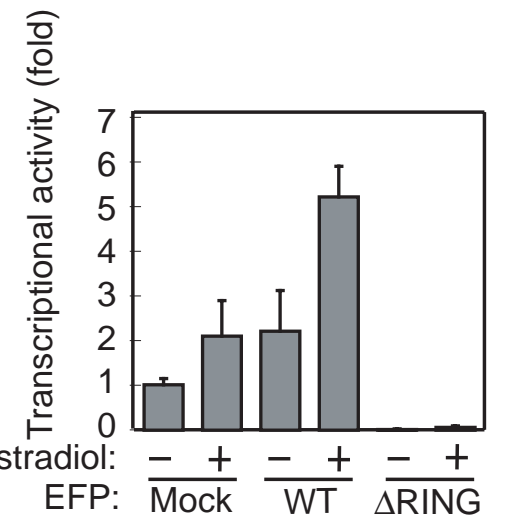

C

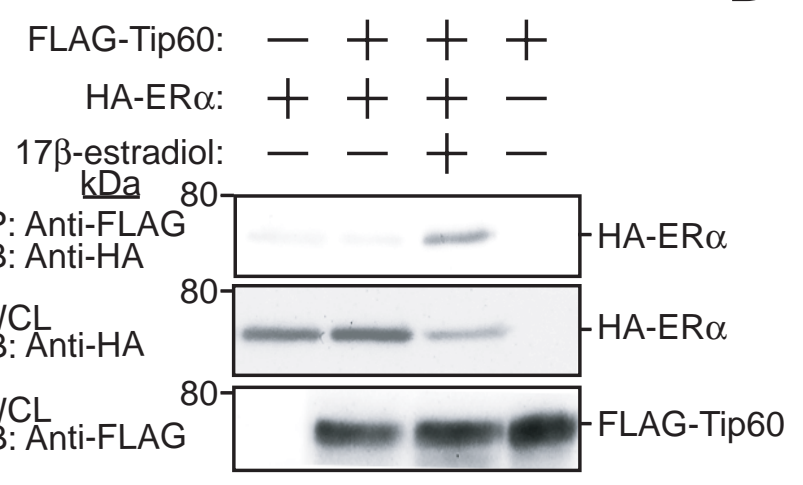

B 흥

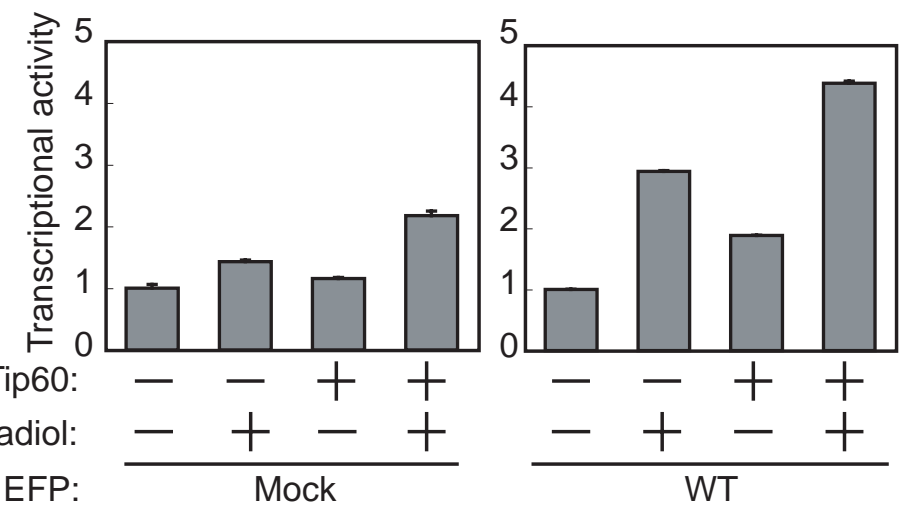

EFP:

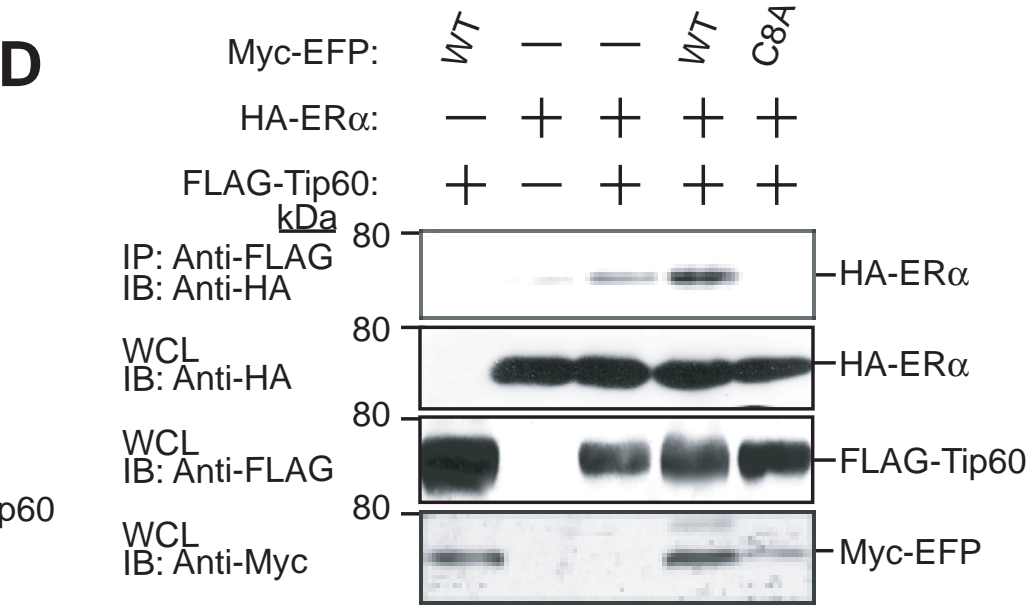

